Slavica

bruxellensia

\section{Slavica bruxellensia}

Revue polyphonique de littérature, culture et histoire

slaves

$11 \mid 2015$

Littérature et philosophie

\title{
Witkacy, un artiste et un philosophe moderne face à des dilemmes post-modernes
}

\section{Teresa Pękala}

Traducteur : Jeremy Lambert

\section{OpenEdition \\ Journals}

Édition électronique

URL : http://journals.openedition.org/slavica/1735

DOI : 10.4000/slavica. 1735

ISSN : 2034-6395

\section{Éditeur}

Université libre de Bruxelles - ULB

\section{Référence électronique}

Teresa Pękala, «Witkacy, un artiste et un philosophe moderne face à des dilemmes post-modernes », Slavica bruxellensia [En ligne], 11 | 2015, mis en ligne le, consulté le 19 avril 2019. URL : http:// journals.openedition.org/slavica/1735; DOI : 10.4000/slavica.1735

Ce document a été généré automatiquement le 19 avril 2019.

\section{c)}

Les contenus de Slavica bruxellensia sont mis à disposition selon les termes de la Licence Creative Commons Attribution - Pas d'Utilisation Commerciale - Pas de Modification 3.0 France. 


\title{
Witkacy, un artiste et un philosophe moderne face à des dilemmes post- modernes
}

\author{
Teresa Pękala \\ Traduction : Jeremy Lambert
}

1 L'histoire de l'art est l'histoire des artistes et des œuvres qui ont perdurés. Cette assertion évidente se répète dernièrement assez souvent sous l'influence d'une nouvelle historiographie qui concentre son attention sur les «petites narrations» historiques et rappelle la possibilité des histoires alternatives. Les œuvres oubliées, qui existent en marge des grands styles artistiques, éveillent un intérêt général. On recherche des trames secondaires dans les biographies des créateurs reconnus, des trames qui n'ont pas été épuisées ou qui ont été un tant soit peu rejetées par la critique. Comme si perpendiculairement aux tendances contemporaines de interprétation, se situait l'histoire des artistes et des penseurs qui se sont inscrits dans l'histoire mais qui, dans leur création, n'ont pas intégré les schémas et les styles académiques obligés. Ils ont dépassé le cadre de leurs disciplines propres, ils ont acquis une expérience artistique, preuve scientifique à l'appui, ainsi qu'un style d'écriture littéraire mais ils se revendiquent de théories scientifiques dans leurs œuvres artistiques. S'ils n'ont pas toujours laissé derrière eux des continuateurs, ils ont acquis de nombreux commentateurs. Ainsi, sans égard pour une conception de l'histoire en particulier ni pour les tendances actuelles d'élargir les limites de celle-ci, l'art et l'esthétique sont, bon gré mal gré, reconnus à travers de grands noms. Aujourd'hui comme hier, l'absence de personnages marquants, peut être interprétée comme le signe d'une situation difficile d'un domaine donné.

2 En 2014, on a fêté le 75e anniversaire de la mort tragique de Stanisław Ignacy Witkiewicz tandis qu'en 2015 , on fête le $130^{\mathrm{e}}$ anniversaire de la naissance de ce célèbre artiste polonais, philosophe, écrivain et dramaturge. Ces anniversaires sont l'occasion de lui rendre hommage, mais ils nous poussent avant tout à réfléchir à l'actualité des œuvres qu'il laissa derrière lui. Le processus de réception de l'œuvre de Witkacy dans la 
conscience de la génération actuelle est également intéressant parce que le créateur polonais est considéré comme un grand visionnaire qui a annoncé de manière très juste des changements radicaux dans la culture, l'art et la philosophie. Comment la génération actuelle jauge-t-elle ce qu'il avait annoncé dans Niemyte dusze (Les Âmes mal lavées), à savoir la «fin de la religion », le «suicide de la philosophie » et le «déclin de l'art»? Quelles conclusions doivent être tirées par rapport à ces visions apocalyptiques dans la perspective d'une modernité tardive, définie comme une époque posthistorique? Peutêtre cela vaut-il la peine d'utiliser dans les recherches de nouveaux outils d'étude, fournis par l'historiographie contemporaine mentionnée plus avant et regarder l'œuvre de Witkiewicz comme un récit construit par un grand maître du geste et de la pause ? Vus par le prisme du théâtre de la vie, sa vie et son œuvre prennent une signification assez symbolique tandis que son suicide en 1939 ne semble être que la conséquence des tragiques désillusions successives suscitées par l'art comme par la culture occidentale tendant vers le déclin. Comme l'écrit l'un de ses commentateurs: "En se suicidant, Witkacy s'est écoulé - sous le poids de sa propre création bien sûr, mais également sous celui du regard, au sens large, qu'il portait sur la réalité et sur son lien (sa relation) avec elle. $»^{1}$ Comment concilier le prophétisme de Witkacy avec l'attitude de constructivisme dynamique visible dans l'exploration de toujours plus de domaines de la réalité?

3 Sa vie et son œuvre appartiennent-elles à ces tentatives de "sauvetage de la modernité ", décrites par Jürgen Habermas? Ou peut-être la comparaison avec les tentatives de sauvetage de «l'habitude de la modernité ", telles que développées par Odo Marquard, est-elle plus pertinente ? Finalement la question qui nous est posée est de savoir si nous avons affaire à un créateur qui se mesure aux « mythes de la modernité » en philosophie ou en art, ou peut-être dans les deux domaines en même temps?

4 Stanisław Ignacy Witkiewicz, appelé Witkacy, a durant de nombreuses années assuré à l'esthétique polonaise une place indéniable parmi les tentatives les plus modernes de l'esthétique occidentale. Est-ce à juste titre? En reconnaissant la haute position de Witkacy dans l'esthétique, nous en remettons-nous aux opinions des philosophes, des artistes, des théâtrologues et des historiens de l'art dont nous faisons nôtres les jugements sur des questions esthétiques ? Ou peut-être à l'inverse est-ce l'appréciation de son système philosohique, de sa philosophie de l'art originale et de sa théorie du théâtre qui attirent notre attention sur son art et nous en suggèrent son appréciation? L'incroyable individualité de l'auteur des Nowe formy $w$ malarstwie ${ }^{2}$ complique la tenue d'études systématiques et la comparaison des résultats des travaux acdémiques des esthètes, des historiens et des philosophes. Cette manière d'agir est condamnée par avance à l'insuccès, tout comme les tentatives qui consistent à isoler la conception esthétique de Witkacy de l'ensemble de son œuvre, tant théorique qu'artistique. Devant l'évidence de l'échec de ceux qui ont tenté de considérer l'œuvre de Witkacy comme un tout cohérent, ne devrions-nous nous contenter de son prophétisme et reconnaître que ce qui est important, ce n'est pas le contenu de ses assertions mais bien le moment de leur expression. Il n'est pas exagéré en effet d'affirmer qu'seuil de la modernité, Witkacy se démarque clairement parmi les autres créateurs par la quantité et le poids des questions qu'il pose au sujet de l'avenir de l'art, de sa place dans la culture, des relations entre la forme et le contenu d'une œuvre, des statuts ontologique et axiologique des valeurs et des expériences esthétiques. Il est un artiste et un philosophe original maniant une langue qui entra durablement dans le discours de la théorie de l'art et de l'esthétique. Il a mis en place tout un dictionnaire de termes, d'expressions et de métaphores concernant l'art 
moderne, au premier lieu desquels sa conception de la Forme Pure. Cependant, lorsque nous nous arrêtons sur le rôle anticipateur des questionnements de Witkacy par rapport au discours contemporain, nous faisons face à une réelle épreuve. Tous les chercheurs ou presque ont évoqué la multiplicité des strates interprétatives et les difficultés qu'ils rencontraient à isoler chez Witkacy le sujet de l'esthétique, et cela fait longtemps déjà que les publications ont dépassé le nombre des deux cents références établies par Lech Sokoł dans les années 1980.

5 L'essence du problème devant lequel nous nous trouvons pourrait conduire à l'idée de l'emprisonnement de l'art par la philosophie telle que l'a formulée Arthur C. Danto ${ }^{3}$. Une analyse plus fine de la relation entre art et philosophie chez Witkacy suggère pourtant qu'est plus adapté le diagnostic de Jozef Tarnowski selon lequel l'emprisonnement de son art et de sa philosophie est réciproque.

6 Sur la base des lettres entre Stanislaw Witkiewicz père et Stanisław Ignacy Witkiewicz, le chercheur pose les questions suivantes :

Witkacy s'est-il développé parallèlement comme philosophe de l'art et comme artiste, ou a-t-il d'abord pris des décisions artistiques et puis seulement cherché des fondements "théoriques", ou, à l'inverse, avait-il des points de vue théoriques sous l'influence desquels il tentait de créer de l'art ?4

Sans entrer dans les détails du débat épistolaire entre le père et le fils étudié par Tarnowski, il s'agit de se rappeler que l'un de leurs points de discorde était le rapport au naturalisme. Selon le chercheur, c'est l'éloignement du naturalisme qui fut la voie qui mena Witkacy à sa propre conception de l'art, conception qui nécessitait un fondement et un complément philosophiques. Witkacy a exposésaconception du nouvel art dans son essai Nowe formy $w$ malarstwie. Dans la réponse à la question du caractère du lien qui unit la cristalisation d'une attitudeartistique aux idées philosophiques, c'est peut-être leur interdépendance qui semble le plus important. Witkacy met en place un nouvel art puis y ajoute des assises philosophiques, la théorie qui en ressort se rapporte ainsi réflexivement sur les décisions artistiques. Une historiosophie assez cohérente en ressort, dans laquelle l'art est traité comme une des formes mouvantes de la culture. D'un autre côté, la vision de l'histoire de l'art telle que la construit Witkiewicz, et qui lui sert à justifier son antinaturalisme, limite considérablement l'objet de l'art à l'art nouveau sur le plan formel. L'art traditionnel basé sur le lien mimétique entre le monde représenté et le monde réel n'est en rien de l'art dans le sens qu'il donnait à ce terme dans sa conception propre. Il justifiait cela par le fait qu'il était convaincu de l'impossibilité d'exprimer des sentiments métaphysiques à un autre moment qu'à celui-là même de la création d'une œuvre d'art, œuvre d'art qui alors avait une valeur novatrice. L'historiosophie créée à l'usage du nouvel art éliminait du champ de l'art une part considérable de la propre création de Witkacy : ses paysages, ses portraits, y compris ceux qui introduisaient une déformation et des éléments de jeu avec les conventions.

7 En se penchant sur le problème moderne de l'interprétation, on remarque également un rapport avec l'esthétique et l'ontologie, oscillant entre la reconnaissance de certains droits objectifs "de la logique du beau», logique "presque aussi rigide qu'une pensée mathématique ", et le subjectivisme esthétique dans lequel la valeur de l'œuvre est soumise entièrement à la capacité d'éveiller chez le récepteur des sentiments métaphysiques.

8 Dans le cadre de l'épineux problème général du lien entre philosophie et art nous rencontrons certaines difficultés lors de l'analyse des rapports des spéculations 
esthétiques avec l'historiosophie et l'ontologie. Considérée comme un tout, la théorie esthétique de Witkacy, avec son interprétation de l'art métaphysique, ses expériences esthétiques et son « contenu formel » de l'œuvre d'art, appartient à la théorie générale de la culture, tout comme la vision «catastrophique» de la chute de l'art, liée à l'affaiblissement des sentiments métaphysiques. Le problème réside en ceci que la manière de considérer l'art comme l'un des domaines de la culture, telle qu'on la rencontre par endroits dans Nowe formy $w$ malarstwie, conduit à d'autres conclusions que lorsque l'on considère les textes dans lesquels il est exclusivement question de l'art pris dans des catégories formelles. C'est unethéorie artistique à part qui traite des questions de détails concernant la composition, la couleur et le style, et prévoit un autre scénario de catastrophe. La chute de l'art est considérée comme une conséquence de l'évolution de sa forme, et la forme doit subir un affaiblissement. L'inassouvissement par la forme est directement liée au temps historique limité, à l'époque moderne, mais le mécanisme de recherche de formes nouvelles et, dans les faits, la limite de leurs combinaisons possibles est un processus immanent.

9 La vision du crépuscule de l'art ne présuppose pas toujours un pronostic catastrophique. Les théoriciens contemporains ont bien compris que la chute de l'art tant annoncée signifie tout simplement un changement dans la manière dont l'art est compris. Les opinions de Danto pourraient en être un exemple de la version optimiste. L'histoire de l'art se réalise par un changement de paradigmes. L'art mimétique est suivi d'une phase expressive qui lui permet de passer ensuite à l'étape de l'art cognitif. A chaque fois, la vision de l'art est soumise à une conception définie de l'histoire. Le temps qui suit la chute de l'art, annoncé comme une catastrophe par Witkacy, pourrait être, selon Danto, un moment heureux dans lequel «nous pouvons faire ce que nous aimons, bien qu'il soit difficile de s'imaginer à quel point le bonheur peut nous rendre heureux $»^{5}$. En annonçant la chute de l'art dans son historiosophie tout en s'affirmant, tant dans sa théorie de l'art que dans son esthétique, en faveur d'un art des formes pures, Witkacy se retrouve au cœur du conflit qui oppose les prémisses traditionnelles de l'art esthétique au nouveau paradigme de l'art non-autonome, intellectuel. Il était l'un des créateurs d'un nouvel art mais utilisait d'anciens instruments conceptuels, empruntés à l'esthétique de son père et, par cette dernière, à des œuvres de nombreux penseurs classiques. La conception de la chute de l'art se rapproche, dans la rhétorique et la résonance idéologique, de la théorie post-avant-gardiste du crépuscule des beaux-arts. L'un des paradoxes de la création de Witkacy était sa volonté de vaincre la conception traditionnelle de l'art, ce qui se marquait clairement dans sa théorie esthétique, tout en maintenant un répertoire complet d'effets sensuels. En témoigne notamment le rôle de la couleur dans ses œuvres, et pas uniquement en peinture, mais également dans son œuvre littéraire. L'effet physique et émotionnel de la couleur fascine Witkacy. Elle remplit le rôle de moyen formel et de symbole enracinés dans l'iconographie : elle crée un espace dans le roman, elle enveloppe les personnages dans les portraits, elle est un élément important de la scénographie dans les drames. La symbolique des couleurs est utilisée de manière particulière pour créer une atmosphère d'irréalité, pour flotter à la frontière entre le rêve et la réalité. Dans ses romans 622 upadki Bunga(Les 622 chutes de Bungo), Nienasycenie( L'Inassouvissement), Pożegnanie jesieni (L'Adieu à l'automne) ${ }^{6}$ on remarque directement un lien avec la tradition de l'impressionnisme et de la peinture stimmung, donc avec un art de l'ambiance, de l'émotion, de l'atmosphère. Ce n'est pas le lieu ici pour spéculer sur l'influence qu'eut la tradition artistique dans l'utilisation de telle ou telle couleur ou dans telle ou telle situation, ni si le fait de peindre remplit une fonction thérapeutique ou 
quand elle fut consciemment un moyen choisi pour rendre un effet spirituel. Il n'en reste pas moins que le choix des couleurs est réalisé magistralement, avec la meilleure intelligence de l'art esthétique. Du reste, la couleur ne disparaît pas des travaux de création dépassant le paradigme esthétique. On pourrait même dire que tout comme dans les œuvres des grands peintres abstraits, elle cesse d'être un moyen pour devenir une valeur esthétique autonome. Même si nous prenons comme préalable qu'en grande partie le travail sur la couleur est uniquement un jeu avec les conventions picturales, le seul fait de s'attarder dans le domaine des conventions usées et critiquées n'est pas sans importance dans l'appréciation de la place de Witkacy sur la scène artistique contemporaine. Dans la perspective du bilan des modifications apportées par l'avantgarde, bilan clos au XXe siècle déjà, je considère positivement cette appréciation, elle témoigne de la forte individualité créative d'un artiste original. Il était critique non seulement à l'égard des formes éculées de l'art naturaliste, mais également à l'égard des modes artistiques qui se multipliaient de son temps ainsi que de la recherche de la nouveauté à tout prix. C'est pourquoi, malgré la tendance générale, je ne considère pas comme fondée l'idée que l'on puisse séparer les paysages et une partie des portraits de Witkacy de l'ensemble de son création. A la lumière des conceptions esthétiques écologiques contemporaines, développées notamment par Arnold Berleant, il faut à nouveau tourner son regard vers l'étape de fascination par les Tatras constitutive, chez lui, de la cristallisation de sa sensibilité esthétique et de son attitude envers la nature. Witkacy remplit avec application la recommandation de son peintre de père qui lui écrivait : « La condition préalable à la réalisation de quoi que ce soit d'artistique, c'est la capacité à regarder la nature. $»^{7}$ « L'œil » sensuel du fils, peintre de son état, a entraîné sa capacité à observer non seulement les détours peints compris dans une perspective large. Witkacy passe le chemin de l'esthétique du beau qui implique une distance à l'esthétique de la sublimation et de l'engagement dans lequel sont inscrits la résistance et la réaction. On voit parfaitement la différence dans le regard sur la nature lorsqu'on compare des photographies de Witkiewicz-père et de Witkacy. Ewa Franczak et Stefan Okołowicz remarquent que dans ses photographies de paysages, Witkacy se concentre sur des fragments de nature éphémères, souvent volatiles et passagers, excluant l'expérience de la contemplation.

Le but de Witkacy n'était pas de réaliser des compositions montrant une image générale de la nature, mais de la reconnaître par l'enregistrement répété et esquissé des phénomènes qui pointent en eux. (...) Le moyen caractéristique qu'avait Witkiewicz de prendre des photographies était de faire des images qui servaient une meilleure reconnaissance de l'existence d'un motif donné. ${ }^{8}$

Ce n'est pas un rejet de l'esthétique sensuelle mais une tentative d'absorption de la beauté de la nature en une métaphysique de la nature présente dans les nombreux courants de l'art contemporain, y compris avant-gardistes.

Il faut se souvenir que l'avant-garde occidentale était divisée en deux courants au moins : d'un côté l'art qui tendait vers une extension maximale de son domaine sans toutefois mettre de côté les valeurs esthétiques et d'un autre l'art anti-esthétique, comme le dadaïsme. S.I.Witkiewicz était un individualiste et même dans sa quête de modernité il suivait d'autres aiguillons que la volonté, générale à cette époque, de s'éloigner de la tradition de l'art académique. La conscience de vider l'art de sa substance et, ressortant de cette conviction, le besoin d'une nouvelle légitimité dans la création artistique le liaient toutefois aux artistes qui lui étaient contemporains. Witkacy essaya de subordonner l'art à la philosophie, ce qui, comme nous l'écrivions plus haut, se cristalisa 
en un lien original et non univoque entre deux domaines de sa création. Un avantgardiste, comme on le sait, n'existe pas sans sa théorie de l'avant-garde. Il en est ainsi de l'art de Witkiewicz qui prend une autre valeur quand nous connaissons son esthétique et sa philosophie. Ce qui ne signifie pas cependant que la première puisse être expliquée par la seconde.

11 A mon sens, rechercher la faiblesse et l'incohérence dans la création artistique de Witkacy par rapport aux fondamentaux d'une théorie artistique générale est un exemple de méprise dans l'interprétation de l'art du $\mathrm{XX}^{\mathrm{e}}$ siècle, surtout de l'époque de la première avant-garde. Cette méprise repose sur la confusion de l'antiesthétisme de certains artistes contemporains avec l'anticallisme. Le philosophe Mieczysław Wallis, spécialiste de l'esthétique, a décrit les arcanes de ce phénomène :

[L'anticallisme est] tourne le dos aux «valeurs esthétiques douces » - au beau (au sens propre), au joli et au charme - et se tourne vers certains types de «valeurs esthétiques extrêmes" - pas seulement vers ce qui est glauque, grotesque, invraissemblable, mais surtout vers ce qui est choquant, agressif, brutal voire même horrible et repoussant. (...) L'horreur, la laideur, l'incroyable, le macabre, employés à titre expressif ou comme caractéristique, n'étaient bien sûr pas étrangers aux artistes du gothique tardif (Matthias Grünewald, Jérôme Bosch), du baroque (Juan de Valdes Leal) ou du symbolisme (Charles Baudelaire, Une charogne). Mais jamais ce cheminement vers ce qui est choquant, agressif ou brutal n'a joué un rôle aussi important que dans l'art moderne, surtout dans l'expressionnisme et le surréalisme. ${ }^{9}$

Wallis propose que soit plutôt utilisé le terme anticallisme pour désigner la majorité des entreprises artistiques contemporains, car il s'agit ici de tourner le dos aux valeurs esthétiques douces - le beau, le charmant, le joli. En comparaison avec les époques précèdentes qui virent également la création d'œuvres esthétiques laides ou grotesques, ce phénomène prend au XX $\mathrm{XX}^{\mathrm{e}}$ siècle une ampleur inégalée. Il s'agit de bien différencier l' anticallisme de l'antiesthétisme programmatique qui, lui, fait l'impasse sur la constitution de valeurs esthétiques en général. Dans les étapes successives de sa création picturale, littéraire et théâtrale, Witkacy est un artiste qui tend vers l'anticallisme, mais comme esthète annonçant l'épuisement de la forme et la chute de l'art, il penche également du côté de l'antiesthétisme. Il n'est cohérent dans aucune de ses aspirations, il est de plus empêtré dans d'inextricables difficultés lorsqu'il s'agit de les concilier. La raison de cet état de fait est à chercher dans l'aspiration qui accompagna Witkacy toute sa vie de donner à son œuvre un caractère de système enraciné dans des bases métaphysiques fortes. La problématique de la métaphysique de Witkacy, qu'il serait trop compliqué d'exposer ici, a toujours inspiré les chercheurs. Certains, notamment Tarnowski, ont décrit les liens qui unissent la philosophie de Witkiewicz à l'hégélianisme. Cette argumentation se doit d'être mentionnée car elle explique en grande partie la manière par laquelle Witkacy comprenait l'essence de la création artistique et ses sources biologique, psychologique et culturelle.

A l'image d'Hegel, Il distingue trois domaines de la culture: l'art, la religion et la philosophie. Pourtant, l'histoire ne se développe pas à travers des formes successives de l'esprit absolu, elle est déterminée par le degré d'apaisement des besoins biologiques et du degré de sociabilisation qui en émane. La source principale de la création est la volonté d'exprimer des sentiments métaphysiques qui diminue à mesure de l'affaiblissement de l'émotivité métaphysique de l'humanité, de plus en plus rassasiée d'un point de vue biologique. Selon l'avis de Witkacy, l'art allait s'effondrer en même temps que la diminution de la sensibilité métaphysique. Les étapes de ce processus de «mort» 
rappellent le passage des beaux-arts à l'anticallisme et plus loin encore, vers des formes qui ne sont déjà plus de l'art dans son sens esthétique classique. C'est ainsi que l'on pourrait interpréter l'état "d'inassouvissement par la forme» quand la seule voie d'expression des sentiments métaphysiques sont les déformations formelles « sauvages ». Wallis a prouvé que la déformation et les valeurs esthétiques extrêmes correspondent à la sensibilité esthétique de l'époque moderne. Witkacy, tout comme Beaudelaire qu'il admirait, n'exclut pas la laideur du canon des valeurs esthétiques positives. Il allait même plus loin en acceptant que la perversion et la déformation fussent une expression artistique de l'art moderne.

Nous comprenons le mot perversion non pas comme les différents démonismes à la Rops, Munch ou Goya, mais plutôt comme la complication et le déséquilibre dans la composition, la disharmonie des couleurs et des tensions directionnelles abstrusesqui mènent à ce qu'on pourrait appeler la « déformation ». (...) Mais n'estce pas justement la richesse des «tensions directionnelles » qui est, à côté de la diversité et du caractère sauvage de la compréhension de la forme, le trait le plus essentiel de l'art d'aujourd'hui ? ${ }^{10}$

Witkacy ne prévoit pourtant pas la possibilité d'un processus infini de modifications formelles dans l'art. En ce sens, il est un pessimiste. Il voit l'étape d'après dans son œuvre, le formisme, comme un pas en direction de la chute inéluctable de toute création artistique et l'une des dernières tentatives d'exprimer des sentiments métaphysiques, " les derniers soubresauts tragiques » de l'art. ${ }^{11}$

Le sentiment nostalgique ou, comme disent certains, tragique de la limite des valeurs générales de la culture occidentale ne signifie pas que la création de Witkacy est crisogène. Ce serait même plutôt l'inverse si nous nous souvenons que dans le geste parodique du rejet, son objet, n'est pas la haute culture des valeurs artistiques et esthétiques, mais bien sa forme diminuée, l'emprise des stéréotypes, la copiedes formes artistiques, le schématisme et la passivité. Dans son article au titre plus qu'évocateur, «Bez Kompromisu » (Sans compromis), Witkacy écrit :

Selon moi, faire un portrait naturaliste, un portrait correct dans un sens psychologique n'est pas condamnable. Même chose pour la photographie, tant bien sûr que cela n'aille pas dire au publicqu'il s'agit là de l'Art le plus élevé, de l'art avec un A majuscule (...). Ce qui est condamnable, c'est la production de mèches naturalistes abjectes, sans aucun besoin fondamental, tout en convainquant le public que le Pure Forme en peinture (...) ce sont des gribouillis sortis tout droit de la tête de personnes qui ne savent pas dessiner. ${ }^{12}$

Dans ces mots résonnent l'amertume et l'expérience ou, pour le dire autrement, la maturité d'un homme de haute culture occidentale. Aujourd'hui, nous dirions sûrement que c'est l'expression d'une conscience postmoderne, qui ne se détache en rien de la culture moderne de l'occident mais se distancie de ses abus. On pourrait en trouver tant, de cestraces de postmodernité, qu'on pourrait en écrire une étude complète. Citons quelques-uns seulement de ces traits caractéristiques de l'art contemporain: l'intertextualité, le pastiche, la théâtralisation de la réalité, le contentement de la copie, de la série, des masques. Grâce à son lien dynamique à la philosophie et à l'art, l'œuvre de Witkacy tend en direction du méta-art et du méta-discours : le commentaire et la distance sont omniprésents. Ils ne sont pourtant pas un simple (dans le sens dans lequel on définit actuellement ce type de démarche artistique) éloignement du réalisme et de représentativité. L'artiste polonais ne s'en défait pas complétement et dans la bouche du héros principal des 622 upadki Bunga, il met des mots qui permettent de «libérer les valeurs ", sous lesquelles il signe en auteur qui s'occupe "de l'obligation de se corriger 
dans le monde réel ». Cette stratégie postmoderne au seuil de la modernité à de quoi étonner. Witkiewicz soumet à la réinterprétation des visions de la culture et des théories de l'art modernistes (mais qui plonge toutefois leurs racines dans le romantisme), tout en imaginant leur dénaturation. Il n'est plus possible de considérer ses décisions artistiques et philosophiquesà l'aune de dualismes modernistes : beau contre laid, bien contre mal, modernité contre tradition, ou même être contre non-être. Nous avons plutôt affaire à une remise en cause de l'évidence et à une recherche d'état intermédiaire " entre » ce qui est réel et ce qui ne l'est pas. Les héros s'expriment avec ironie sur les questions philosophiques au sujet de la désillusion de ce que l'on ne peut pas parler de soi en "je » et se satisfaire du néant.

Dans d'autres travaux, j'ai nommé la personnalité littéraire de Witkiewicz dans ses drames optique infantile: elle modifie les proportions naturelles mais en même temps elle se permet un engagement dans le monde joué « pour de faux $»^{13}$. J'irais chercher entre autres l'orginalité de la pensée de Witkacy dans le caractère de son discours. Tant en art qu'en philosophie, nous avons affaire à un effet de "suspension" de la véracité des contenus présentés à l'aide d'un méta-discours. En peinture et dans les drames, cette fonction est remplie par la Forme Pure, mais en théorie - au concept de construction. La création artistique est une construction et même dans les trames qui semblent métaphysiques, Witkacy s'en souvient sans cérémonie. Cette manière de procéder a de grands prédécesseurs dont la lecture a éduqué Witkacy : Charles Baudelaire ou Friedrich Wilhelm Joseph Schelling. A leur suite, il tente d'atteindre le sens métaphysique de la construction. Construire une œuvre d'art, c'est construire un système métaphysique, l'artiste est un philosophe qui tente de savoir comment atteindre dans les formes de l'art historique l'espression d'un tout et de l'infinité du monde absolu. L'artiste pour lequel l'acte de création est un acte intellectuel ici et maintenant rappelle au récepteur que l'art n'est pas une expression vivante et l'expression spontanée d'émotions accidentelles.

La distance et le commentaire, l'ironie et l'auto-ironie sont omniprésents, nous le répétons. C'est un jeu avec le monde, un jeu pour une sérieuse cagnotte. Dans les fragments de Normalnego człowieka (Un homme normal) qui ont été conservés, l'opposition de l'absurde et du chaos du monde se termine par la ridiculisation du protagoniste principal, toutefois la tentative d'atteindre l'idée d'un bien inconditionnel est remplie d'authenticité. Comme dans une paraphrase de la Grande Improvisation, le Maître ressuscité déplore qu'à nouveau il y ait quelque chose qui fasse qu'on retourne au nonêtre. C'est de la sensibilité du récepteur que dépend si celui-ci ressent de l'angoisse (métaphysique ?) ou s'il sera sujet au pouvoir de l'ironie sous-tendant l'existence de tout fondement permanent de l'existence. Lors de la conférence anniversaire intitulée Witkacy $w$ kontekstach. Stanisław Ignacy Witkiewicz a kryzys metafizyki (Witkacy en contextes. Stanisław Ignacy Witkiewicz et la crise de la métaphysique) tenue du 23 au 25 mai 2014 à Zakopane, le professeur Ewa Łubieniewska a souligné l'épuisementdu potentiel du théâtre moderne dans le remplacement de l'étrangeté witkacéenne par le «bizarre » et de la « forme pure » par la « forme vide ». ${ }^{14}$

17 Afin de ne pas conclure sur une réflexion par trop pessimiste, j'ajoute que l'auteur de cette dernière a écrit un livre conséquent sur le théâtre qui fait perdurer l'héritage de Witkiewicz: le théâtre Witkacy de Zakopane. Sa formule s'inscrit dans l'assemblage witkacéen de conventions théâtrales anciennes et actuelles. La maturité intellectuelle et le caractère génial de l'art de Witkacy trouve un complément dans le théâtre qui ne porte pas son nom par accident ni à cause du genius loci de Zakopane. D'une manière 
extraordinaire, mais également dramatique, l'individualisme non conventionnel de l'artiste a rencontré la vision universelle de la culture occidentale. Le fait que nous prenons « pour jouer » ou « au sérieux » la question de savoir où nous sommes et quelle est notre identité témoigne bien de la condition de cette culture. Et qu'il n'est pas aisé d'y répondre, c'est ce qu'a parfaitement montré, sur un plan purement artistique, le spectacle deFélix Lope de Vega y Carpio intitulé Na niby - naprawdĘ (pour de rire - pour de vrai) interprétée par la troupe du Théâtre Witkacy dans une mise en scène d'Andrzej Dziuk. La tragicomédie du dramaturge espagnol introduit naturellement dans le climat de discussions sur le relativisme de la vie de l'homme et le caractère indéfini des limites entre les différents champs de la réalité. De notre réalité, 130 ans après la naissance de Witkiewicz, à la frontière (peut-être) de nouveaux paradigmes culturels.

\section{NOTES}

1. Ostowski A., « Nicość - pro et contra » (Le rien - pro et contra), in : Przyszłość Witkacego, sous la direction de Pękala T., Universitas, Cracovie, 2010, p. 58.

2. Witkiewicz St. I., Nowe formy $w$ malarstwie $i$ wynikajĄce stĄd nieporozumienia. Szkice estetyczne, édité par Degler J. \& Sokół L., PIW, Varsovie, 2002. Edition française: Les formes nouvelles en peinture et les malentendus qui en découlent, traduit du polonais par Antoine Baudin, L'Age d'Homme, coll. Slavica - Ecrits sur l'art, Lausanne, 1979.

3. Danto A. C., "Philosophical Coertion of Art» (La coercition philosophique de l'art), in: Proceedings of the Montreal Congress - 1984 (Actes du Congrès de Montréal). Edition utilisée ici : «Filozoficzne zniewolenie sztuki», traduction polonais de Marek Janiak, in : Studia Estetyczne, t. XXII, 1986-1990.

4. Tarnowski J.,« Wzajemne zniewolenie filozofii i sztuki » (L'emprisonnement réciproque de la philosophie et de l'art), in : Przyszłość Witkacego, op. cit., p. 89.

5. Danto A.C., «Koniec sztuki », in: Danto A.C., Świat sztuki (Le monde de l'art), traduit de l'anglais vers le polonais par L. Sosnowski, Wydawnictwo Uniwersytetu Jagiellońskiego, Cracovie, 2006, pp. 214-215.

6. Les trois ouvrages sont parus en français aux Editions L'Âge d'Homme (Lausanne) dans la traduction d'Alain Van Crugten. [NdlR]

7. Witkiewicz S. I., Sztuka i krytyka u nas (L'art et la critique chez nous), t. 1, Varsovie, 1949, p. 76.

8. Franczak E. \& Okołowicz S., Przeciw nicości. Fotografie Stanisława Ignacego Witkiewicza (Contre le néant. Photographies de Stanisław Ignacy Witkiewicz), Cracovie, 1986, pp. 19-21.

9. Wallis M., Wybór pism estetycznych (Choix d'œuvres esthétiques), textes introduit, choisis et mis en forme par PĘkala T., Universitas, Cracovie, 2004, p. 215.

10. Witkiewicz S. I., Nowe formy $w$ malarstwie. Szkice estetyczne. Teatr (Les formes nouvelles en peinture. Essais esthétiques. Théâtre), Varsovie, 1974, p. 223.

11. Witkiewicz S. I., O znaczeniu filozofii dla krytyki i inne artykuły polemiczne (Sur la signification de la philosophie pour la critique et autres articles polémiques), textes mis en forme par Leszczyński J., avec une postface de Dziemidok B., PWN, Varsovie, 1976, p. 142.

12. Witkiewicz S. I., "Bez kompromisu », in : Polska Zbrojna, 1931. Voir l'interprétation de Anna Jamroziakowa de la relation de Witkacy à l'art sur la base de ce fragment: Jamroziakowa A, «Kobieca podmiotowość w symbolicznym porzĄdku?» (l'objectivité féminine dans un ordre 
symbolique? ", in : Powroty do Witkacego. Materiały sesji naukowej poświĘconej Stanisławowi Ignacemu Witkiewiczowi. Stupsk, 7-9 maja 2004 (Les retours de Witkacy. Matériel de la séance académique consacrée à Stanisław Ignacy Witkiewicz. Słupsk, 7-9 mai 2004), sous la direction de Tarnowskiego J., Muzeum Pomorza Środkowo w Słupsku, Słupsk, 2006, pp. 81-82.

13. Voir PĘkala T.« Witkacy kontra Witkiewicz - konflikt paradygmatów », in: Powroty do Witkacego, ibid., pp. 225-239.

14. La réflexion de Ewa Łubieniewska est à retrouver dans Witkacy w kontekstach. Stanisław Ignacy Witkiewicz a kryzys metafizyki (Witkacy en contextes. Stanisław Ignacy Witkiewicz et la crise de la métaphysique), sous la direction de PĘkala T., Wyd. UMCS, Lublin, 2015 (en préparation).

\section{INDEX}

Index géographique : Pologne

oeuvretraite Witkiewicz St. I. : Niemyte dusze, Witkiewicz St. I. : Nowe formy w malarstwie, Witkiewicz St. I. : 622 upadki Bunga, Witkiewicz St. I. : Nienasycenie, Witkiewicz St. I. :

Pożegnanie jesieni, Witkiewicz St. I. : Normalnego człowieka

Mots-clés : littérature polonaise, philosophie polonaise, esthétique

\section{AUTEURS}

\section{TERESA PĘKALA}

Doyen de la Faculté de Philosophie et Sociologie de l'Université Marie Curie-Skłodowska de Lublin, directrice du Département d'Esthétique 